\title{
Taxonomy of Alteromonas: A. nigrifaciens sp. nov., nom. rev.; A. macleodii; and A. haloplanktis
}

\author{
PAUL BAUMANN,${ }^{1 *}$ LINDA BAUMANN,${ }^{1}$ RON D. BOWDITCH,${ }^{1}$ AND BLAINE BEAMAN ${ }^{2}$ \\ Department of Bacteriology, University of California, ${ }^{1}$ and Department of Medical Microbiology, University of California \\ Medical School, ${ }^{2}$ Davis, California 95616
}

\begin{abstract}
On the basis of phenotypic properties and the guanine-plus-cytosine content of the deoxyribonucleic acid, as well as the immunological relationship of the glutamine synthetase and the iron-containing superoxide dismutase, strain $217^{\mathrm{T}}$ (= ATCC 19375) (T = type strain), which was previously designated "Pseudomonas nigrifaciens," was shown to be a member of a distinct species in the genus Alteromonas. Since this species designation was not included on the Approved Lists of Bacterial Names, it has been revived as Alteromonas nigrifaciens sp. nov., nom. rev., with type strain 217 (= ATCC 19375). The phenotypic properties of strains of Alteromonas macleodii and Alteromonas haloplanktis are also described.
\end{abstract}

In 1940 White described a bacterium which caused discoloration of mildly salted butter (7). This organism was straight, polarly flagellated, and rod shaped, grew at $4^{\circ} \mathrm{C}$, required $\mathrm{NaCl}$, and produced a brown or black pigment. White suggested that it be named "Pseudomonas nigrifaciens." Subsequently, Norton and Jones (4) isolated a pigmented marine strain which they equated with this species. In our studies of nonfermentative marine bacteria, we obtained a number of isolates having similar pigmentation (1). Characterization of these strains, as well as " $P$. nigrifaciens" strain $217^{\mathrm{T}}\left(=\right.$ ATCC $\left.19375^{\mathrm{T}}\right)(\mathrm{T}=$ type strain $)$, showed that they belong to different genera (1). In addition, the many differences between the phenotype of the " $P$. nigrifaciens" strain and the published phenotype of the isolate of Norton and Jones (4) indicated that these two strains could not be assigned to the same species. On the basis of the guanine-plus-cytosine $(\mathrm{G}+\mathrm{C})$ content of its deoxyribonucleic acid (DNA) (42.9 mol\%) and its general phenotypic properties, "P. nigrifaciens" was shown to be a member of the newly proposed genus Alteromonas in 1972 (1). This species is not on the Approved Lists of Bacterial Names (6) and is listed as species incertae sedis in the chapter on Alteromonas in Bergey's Manual of Systematic Bacteriology, 9th ed. (P. Baumann, M. J. Gauthier, and L. Baumann, in press). A recent study of the relationships of the glutamine synthetases and iron-containing superoxide dismutases from species of Alteromonas indicated that " $P$. nigrifaciens" is closely related to Alteromonas haloplanktis, Alteromonas espejiana, and Alteromonas undina (E. F. DeLong, L. Baumann, R. D. Bowditch, and P. Baumann, Arch. Microbiol., in press). In this paper we revive this species under the designation Alteromonas nigrifaciens $\mathrm{sp}$. nov., nom. rev. and provide a phenotypic description of the sole available strain of this species, as well as descriptions of Alteromonas macleodii and $A$. haloplanktis.

\section{MATERIALS AND METHODS}

"P. nigrifaciens" strain $217^{\mathrm{T}}\left(=\right.$ ATCC $\left.19375^{\mathrm{T}}\right)$, the original isolate of White (7), was obtained from the American Type Culture Collection. The sources of the remaining strains used in this study, as well as the methods used for isolation, have been described previously $(1,3)$. Detailed protocols of the methods used for phenotypic characterization of the strains have also been published previously (2).

\footnotetext{
* Corresponding author.
}

The nutritional spectrum of " $P$. nigrifaciens" was determined in media containing $0.01 \%$ (wt/vol) yeast extract (2).

\section{RESULTS}

All of the strains of "P. nigrifaciens," A. macleodii, and A. haloplanktis were straight rod-shaped organisms which were motile by means of polar flagella. Figure 1 is an electron micrograph showing the general cell morphology and flagellation of " $P$. nigrifaciens." Figure 2 shows the colonial morphology and pigmentation of strain $217^{\mathrm{T}}$ grown at different temperatures. Electron and light micrographs of $A$. macleodii and $A$. haloplanktis have been published elsewhere (5; Baumann, Gauthier, and Baumann, in press). All strains of these three species utilized D-glucose, maltose, acetate, propionate, and pyruvate as sole or principal sources of carbon and energy. All grew at $30^{\circ} \mathrm{C}$, produced an extracellular lipase, were oxidase positive, and required $\mathrm{Na}^{+}$or seawater for growth.

None of the strains was able to denitrify or to utilize $\mathrm{H}_{2}$ as an energy source and $\mathrm{CO}_{2}$ as a carbon source. None grew at $45^{\circ} \mathrm{C}$, accumulated poly- $\beta$-hydroxybutyrate as an intracellular reserve product, or had a constitutive arginine dihydrolase system. The following compounds were not utilized by any of the strains: D-ribose, D-arabinose, D-fucose, $\mathrm{L}$-rhamnose, inulin, saccharate, mucate, glucuronate, formate, oxalate, malonate, maleate, glutarate, adipate, pimelate, suberate, azelate, sebacate, DL-malate, D-(-)-tartrate, L-(+)tartrate, meso-tartrate, $\alpha$-ketoglutarate, laevulinate, citraconate, itaconate, mesaconate, erythritol, sorbitol, meso-inositol, adonitol, ethylene glycol, 2,3-butylene glycol, methanol, isopropanol, isobutanol, D-mandelate, L-mandelate, benzoyl formate, benzoate, $o$-hydroxybenzoate, $m$ hydroxybenzoate, $p$-hydroxybenzoate, phenylacetate, quinate, $\mathrm{D}$ - $\alpha$-alanine, $\beta$-alanine, $\mathrm{L}$-norleucine, $\gamma$-aminobutyrate, $\delta$-aminovalerate, L-tryptophane, D-tryptophane, DL-kynurenine, kynurenate, anthranilate, $m$-aminobenzoate, $p$-aminobenzoate, methylamine, ethanolamine, benzylamine, putrescine, spermine, histamine, tryptamine, butylamine, 2amylamine, phentylamine, betaine, sarcosine, creatine, pantothenate, acetamide, nicotinate, nicotinamide, trigonelline, allantoin, adenine, guanine, cytosine, thymine, uracil, and $n$-hexadecane. The traits present in only some of the strains are shown in Table 1.

\section{DISCUSSION}

The genus Alteromonas as currently defined (Baumann, Gauthier, and Baumann, in press) consists of gram-negative, 


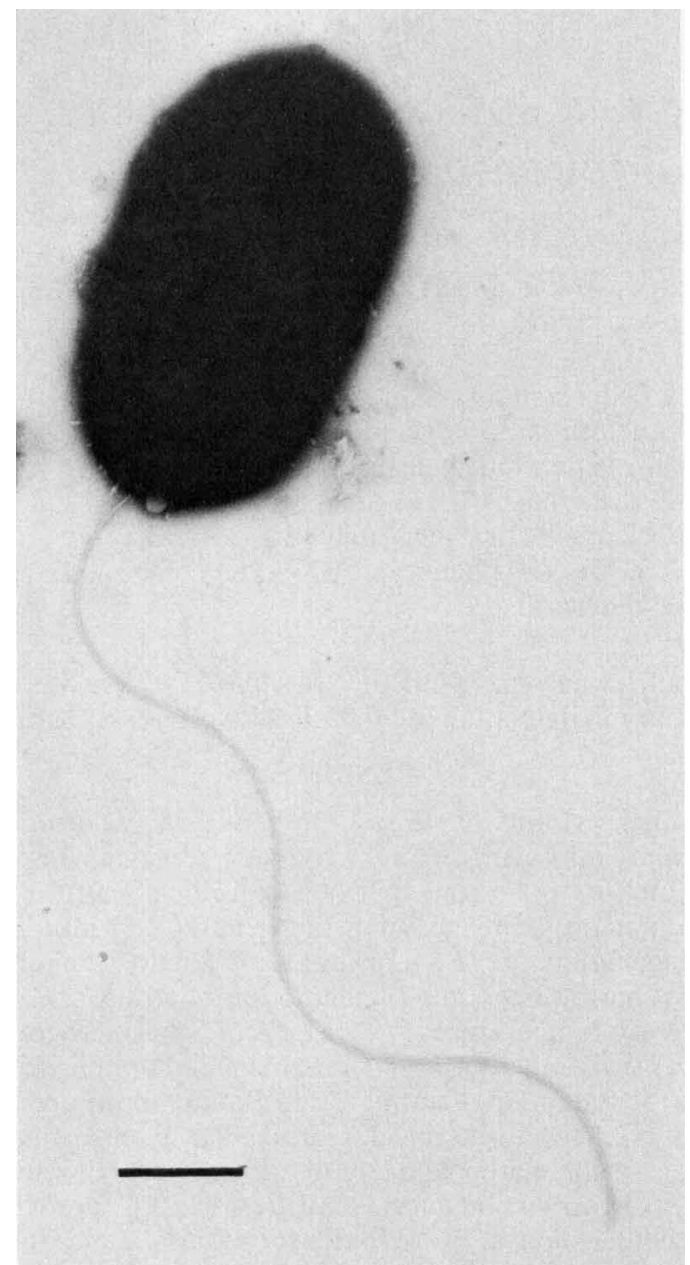

FIG. 1. Electron micrograph of A. nigrifaciens $217^{\mathrm{T}}$ in the exponential phase of growth in liquid medium. $\times 24,000 . \mathrm{Bar}=0.5 \mu \mathrm{m}$.

polarly flagellated, rod-shaped organisms which do not accumulate poly- $\beta$-hydroxybutyrate as an intracellular reserve product. These bacteria are chemoorganotrophs that are capable of respiratory but not fermentative metabolism, with molecular oxygen as the universal electron acceptor. None denitrifies or has a constitutive arginine dihydrolase system. All require $\mathrm{Na}^{+}$or a seawater base for growth. The $\mathrm{G}+\mathrm{C}$ contents of the DNAs range from 38 to $50 \mathrm{~mol} \%$. " $P$. nigrifaciens" strain $217^{\mathrm{T}}$ (= ATCC $19375^{\mathrm{T}}$ ) has all of these properties and is therefore assigned to the genus Alteromonas. The following selected phenotypic properties distinguish strain $217^{\mathrm{T}}$ from all of the previously described species of Alteromonas by 6 to 11 traits: production of a brown or black pigment; ability to utilize $\mathrm{D}$-fructose, maltose, succinate, fumarate, citrate, and glycerol; and inability to produce an extracellular amylase or utilize D-mannose, sucrose, $\mathrm{N}$ acetylglucosamine, erythritol, and D-sorbitol. These findings, together with the immunological studies of the ironcontaining superoxide dismutases and glutamine synthetases of Alteromonas (DeLong et al., in press), indicate that strain $217^{\mathrm{T}}$ constitutes a distinct species, which we designate $A$. nigrifaciens sp. nov., nom. rev.

Below is a description of $A$. nigrifaciens, as well as descriptions of $A$. macleodii and $A$. haloplanktis. In our previous publications $(1,3,5)$ we did not include the phenotypic properties of the type strain of $A$. macleodii or the properties of the remaining strains of the latter two species.

Description of Alteromonas nigrifaciens. Alteromonas nigrifaciens sp. nov., nom. rev. (ni.gri.fa'ci.ens. L. adj. niger black; L. v. facio to make; M.L. part. adj. nigrofaciens blackening) cells are straight rods 0.8 to $1.2 \mu \mathrm{m}$ wide and 1.8 to $2.3 \mu \mathrm{m}$ long and are motile by single flagella at one pole (Fig. 1). Grows at 4 to $30^{\circ} \mathrm{C}$ but not at $35^{\circ} \mathrm{C}$. Produces a brown or black pigment which is especially prominent at lower temperatures (Fig. 2). Produces an extracellular gelatinase and lipase but not an amylase, alginase, or chitinase. Utilizes 26 organic compounds, including hexoses, disaccharides, fatty acids, tricarboxylic acid cycle intermediates, alcohols, and amino acids. The $\mathrm{G}+\mathrm{C}$ content of the DNA is $42.9 \mathrm{~mol} \%$.

The type strain is strain 217 (= ATCC 19375).

Description of $\boldsymbol{A}$. macleodii. Straight rods 0.9 to $1.3 \mu \mathrm{m}$ wide and 1.8 to $2.5 \mu \mathrm{m}$ long that are motile by single flagella at one pole. Produces an extracellular amylase, gelatinase, and lipase but not a chitinase. Utilizes 26 to 44 organic compounds, including hexoses, disaccharides, sugar acids, and amino acids. The $\mathrm{G}+\mathrm{C}$ content of the DNA is 44.9 to $46.4 \mathrm{~mol} \%$.

The type strain is strain 107 (= ATCC 27126).

Description of $\boldsymbol{A}$. haloplanktis. Straight rods 0.8 to $1.2 \mu \mathrm{m}$ wide and 1.9 to $3.0 \mu \mathrm{m}$ long that are motile by single flagella at one pole. Produces an extracellular gelatinase and lipase but not an alginase. Utilizes 20 to 38 organic compounds, including hexoses, disaccharides, fatty acids, tricarboxylic
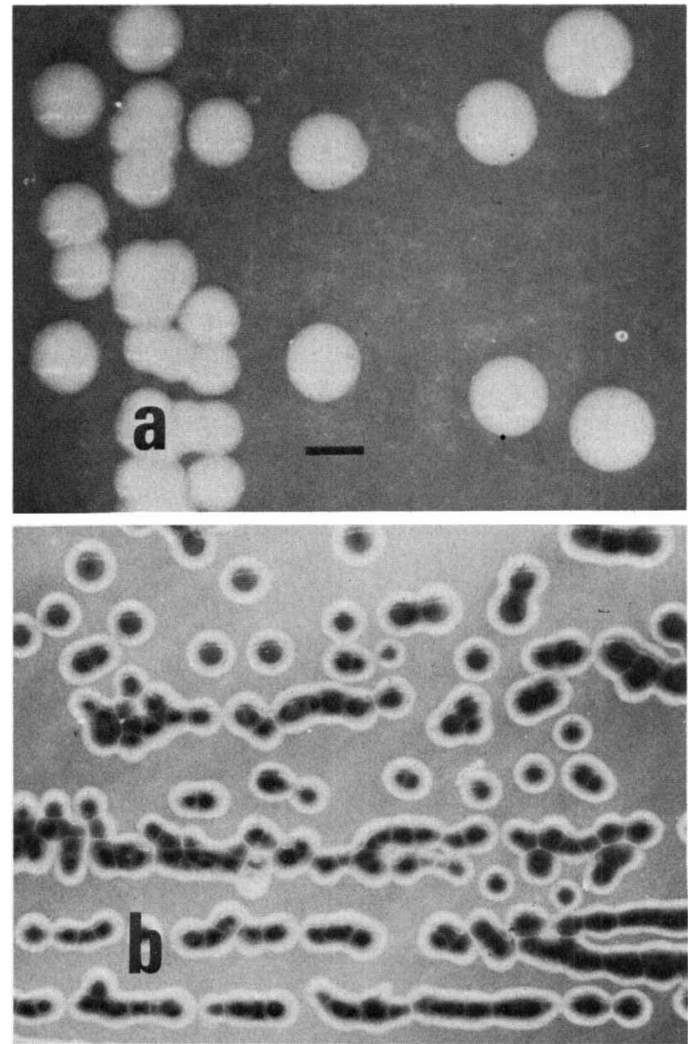

FIG. 2. Colony morphology and pigmentation of $A$. nigrifaciens grown on complex medium for 2 days at $30^{\circ} \mathrm{C}$ (a) and for 3 days at $4^{\circ} \mathrm{C}$ (b). Bar $=2 \mathrm{~mm}$. 
TABLE 1. Phenotypic properties not universally present in A. nigrifaciens, A. macleodii, and A. haloplanktis ${ }^{a}$

\begin{tabular}{|c|c|c|c|c|c|c|c|c|c|}
\hline \multirow[b]{2}{*}{ Property } & \multirow{2}{*}{$\begin{array}{l}\text { Phenotype of } \\
\text { A. nigrifaciens } \\
\text { strain } 217^{\mathrm{T}} \\
(=\mathrm{ATCC} \\
\left.19375^{\mathrm{T}}\right)\end{array}$} & \multicolumn{4}{|c|}{ A. macleodii } & \multicolumn{4}{|c|}{ A. haloplanktis } \\
\hline & & $\begin{array}{l}\text { No. of } \\
\text { strains } \\
\text { positive } \\
(n=21)\end{array}$ & $\begin{array}{c}\text { Phenotype of } \\
\text { strain } 107^{\mathrm{T}} \\
(=\text { ATCC } \\
\left.27126^{\mathrm{T}}\right)\end{array}$ & $\begin{array}{l}\text { Less } \\
\text { common } \\
\text { pheno- } \\
\text { type }\end{array}$ & $\begin{array}{l}\text { Strain(s) with less } \\
\text { common } \\
\text { phenotype }\end{array}$ & $\begin{array}{l}\text { No. of } \\
\text { strains } \\
\text { positive } \\
(n=23)\end{array}$ & $\begin{array}{c}\text { Phenotype of } \\
\text { strain } 215^{\mathrm{T}} \\
(=\text { ATCC } \\
\left.14393^{\mathrm{T}}\right)\end{array}$ & $\begin{array}{l}\text { Less } \\
\text { common } \\
\text { pheno- } \\
\text { type }\end{array}$ & $\begin{array}{l}\text { Strain(s) with less } \\
\text { common } \\
\text { phenotype }\end{array}$ \\
\hline $\mathrm{NO}_{3}{ }^{-} \rightarrow \mathrm{NO}_{2}^{-}$ & - & 2 & - & + & 103,106 & 9 & - & + & $\begin{array}{c}119-121,124, \\
126,131 \\
318-320\end{array}$ \\
\hline $\begin{array}{l}\text { Amino acid re- } \\
\text { quirement }\end{array}$ & + & 0 & - & & & 7 & - & + & $214,318-323$ \\
\hline \multicolumn{10}{|l|}{ Growth at: } \\
\hline $4^{\circ} \mathrm{C}$ & + & 0 & - & & & 2 & - & + & 214,216 \\
\hline $35^{\circ} \mathrm{C}$ & - & $21^{b}$ & + & & & $21^{b}$ & - & - & $214,215^{\mathrm{T}}$ \\
\hline $40^{\circ} \mathrm{C}$ & - & 15 & + & - & $\begin{array}{c}98,99,103- \\
105,118\end{array}$ & 0 & - & & \\
\hline \multicolumn{10}{|l|}{ Production of: } \\
\hline Amylase & - & $19^{b}$ & + & - & 106,118 & 3 & - & + & $121,322,323$ \\
\hline Gelatinase & + & $20^{b}$ & + & - & 106 & $23^{b}$ & + & & \\
\hline Alginase & - & 3 & + & + & $98,99,107^{\mathrm{T}}$ & 0 & - & & \\
\hline Chitinase & - & 0 & - & & & 14 & - & - & $\begin{array}{l}214,215^{\mathrm{T}}, 216 \\
\quad 318-323\end{array}$ \\
\hline \multicolumn{10}{|l|}{ Utilization of: } \\
\hline D-Xylose & - & 4 & + & + & $\begin{array}{l}98,107^{\mathrm{T}}, 116, \\
117\end{array}$ & 1 & - & + & 122 \\
\hline L-Arabinose & - & 0 & - & & & 15 & - & - & $\begin{array}{l}119,121,126 \\
214,215^{\mathrm{T}} \\
216,322,323\end{array}$ \\
\hline L-Rhamnose & - & 1 & - & + & 116 & 2 & - & + & 122,125 \\
\hline D-Mannose & - & 0 & - & & & $21^{b}$ & - & - & $215^{\mathrm{T}}, 216$ \\
\hline D-Galactose & + & $21^{b}$ & + & & & 5 & - & + & $\begin{array}{c}120,121,127 \\
128,214\end{array}$ \\
\hline D-Fructose & + & $21^{b}$ & + & & & 15 & + & - & $\begin{array}{c}119-121,124 \\
126,216 \\
322,323\end{array}$ \\
\hline Sucrose & - & $21^{b}$ & + & & & $21^{b}$ & - & - & $214,215^{\mathrm{T}}$ \\
\hline Trehalose & - & $19^{b}$ & + & - & 109,110 & $19^{b}$ & - & - & $\begin{array}{l}120,214,215^{\mathrm{T}}, \\
216\end{array}$ \\
\hline Cellobiose & - & $21^{b}$ & + & & & 0 & - & & \\
\hline Melibiose & + & $20^{b}$ & + & - & 109 & 0 & - & & \\
\hline Lactose & + & $21^{b}$ & + & & & 2 & - & + & 127,128 \\
\hline Salicin & - & $21^{b}$ & + & & & 0 & - & & \\
\hline Gluconate & + & $18^{b}$ & + & - & $102,106,108$ & 1 & - & + & 214 \\
\hline Galacturonate & - & $18^{b}$ & + & - & $102,103,118$ & 1 & - & + & 130 \\
\hline $\begin{array}{l}N \text {-acetylglu- } \\
\text { cosamine }\end{array}$ & - & 7 & + & + & $\begin{array}{c}98,99,101 \\
107^{\mathrm{T}}, 108 \\
113,116\end{array}$ & $21^{b}$ & - & - & $215^{\mathrm{T}}, 216$ \\
\hline Butyrate & + & $21^{b}$ & + & & & 12 & - & - & $\begin{array}{l}121,123-125, \\
\quad 127-132,215^{\mathrm{T}}\end{array}$ \\
\hline Isobutyrate & + & $21^{b}$ & + & & & $20^{b}$ & - & - & $121,215^{\mathrm{T}}, 216$ \\
\hline Valerate & + & $21^{b}$ & + & & & 17 & + & - & $\begin{array}{l}123-126,130 \\
132\end{array}$ \\
\hline Isovalerate & - & 5 & - & + & $\begin{array}{l}104-106,109 \\
114\end{array}$ & 1 & - & + & 214 \\
\hline Caproate & + & $19^{b}$ & + & - & 113,117 & 2 & - & + & 214,216 \\
\hline Heptanoate & - & 11 & + & - & $109-118$ & 7 & - & + & $\begin{array}{c}126,129-132 \\
214,216\end{array}$ \\
\hline Caprylate & - & $21^{b}$ & + & & & 18 & + & - & $\begin{array}{c}121,127,128 \\
322,323\end{array}$ \\
\hline Pelargonate & - & $21^{b}$ & + & & & $19^{b}$ & + & - & $\begin{array}{l}121,214,322, \\
323\end{array}$ \\
\hline Caprate & - & $21^{b}$ & + & & & $23^{b}$ & + & & \\
\hline Succinate & + & 0 & - & & & $23^{b}$ & + & & \\
\hline Fumarate & + & 0 & - & & & $23^{b}$ & + & & \\
\hline $\begin{array}{l}\text { DL- } \beta \text {-Hydroxy- } \\
\text { butyrate }\end{array}$ & - & 12 & - & - & $\begin{array}{l}98-104,107^{\mathrm{T}}, \\
108\end{array}$ & 13 & - & - & $\begin{array}{c}120,123-126 \\
130-132 \\
215^{\mathrm{T}}, 216\end{array}$ \\
\hline DL-Lactate & + & 16 & + & - & $100-103,106$ & 2 & - & + & 122,125 \\
\hline DL-Glycerate & - & $21^{b}$ & + & & & 0 & - & & \\
\hline Citrate & + & 0 & - & & & $21^{b}$ & - & - & $215^{\mathrm{T}}, 216$ \\
\hline Aconitate & + & 0 & - & & & $21^{b}$ & - & - & $215^{\mathrm{T}}, 216$ \\
\hline
\end{tabular}


TABLE 1-Continued

\begin{tabular}{|c|c|c|c|c|c|c|c|c|c|}
\hline \multirow[b]{2}{*}{ Property } & \multirow{2}{*}{$\begin{array}{c}\text { Phenotype of } \\
\text { A. nigrifaciens } \\
\text { strain } 217^{\top} \\
(=\text { ATCC } \\
\left.19375^{\top}\right)\end{array}$} & \multicolumn{4}{|c|}{ A. macleodii } & \multicolumn{4}{|c|}{ A. haloplanktis } \\
\hline & & $\begin{array}{l}\text { No. of } \\
\text { strains } \\
\text { positive } \\
(n=21) \\
\end{array}$ & $\begin{array}{c}\text { Phenotype of } \\
\text { strain } 107^{T} \\
(=\text { ATCC } \\
\left.27126^{T}\right)\end{array}$ & $\begin{array}{l}\text { Less } \\
\text { common } \\
\text { pheno- } \\
\text { type }\end{array}$ & $\begin{array}{l}\text { Strain(s) with less } \\
\text { common } \\
\text { phenotype }\end{array}$ & $\begin{array}{l}\text { No. of } \\
\text { strains } \\
\text { positive } \\
(n=23) \\
\end{array}$ & $\begin{array}{c}\text { Phenotype of } \\
\text { strain } 215^{\mathrm{T}} \\
(=\mathrm{ATCC} \\
\left.14393^{\mathrm{T}}\right)\end{array}$ & $\begin{array}{c}\text { Less } \\
\text { common } \\
\text { pheno- } \\
\text { type }\end{array}$ & $\begin{array}{l}\text { Strain(s) with less } \\
\text { common } \\
\text { phenotype }\end{array}$ \\
\hline Mannitol & - & 9 & + & + & $\begin{array}{l}98,99,107^{\mathrm{T}} \\
108,111-113 \\
115,116\end{array}$ & 11 & - & + & $\begin{array}{l}122-124,127 \\
128,131 \\
132,318-321\end{array}$ \\
\hline Glycerol & + & $21^{b}$ & + & & & 0 & - & & \\
\hline Propylene glycol & - & 5 & - & + & $\begin{array}{l}109,110,114- \\
116\end{array}$ & 3 & - & + & $119-121$ \\
\hline Ethanol & + & $17^{b}$ & + & - & $\begin{array}{l}100,101,103 \\
\quad 118\end{array}$ & 18 & - & - & $\begin{array}{l}215^{\mathrm{T}}, 318,319, \\
\quad 322,323\end{array}$ \\
\hline$n$-Propanol & + & 15 & + & - & $\begin{array}{l}100,101,103 \\
\quad 112,113,118\end{array}$ & 15 & - & - & $\begin{array}{l}122,131,132, \\
215^{\mathrm{T}}, 318, \\
319,322,323\end{array}$ \\
\hline Glycine & - & 14 & + & - & $\begin{array}{c}101,102,104 \\
108,111 \\
112,115\end{array}$ & 14 & + & - & $\begin{array}{l}119-123,132, \\
216,322,323\end{array}$ \\
\hline L- $\alpha$-Alanine & + & $19^{b}$ & + & - & 104,110 & $23^{b}$ & + & & \\
\hline L-Serine & + & 9 & - & + & $\begin{array}{c}105,109-112 \\
114-117\end{array}$ & $23^{b}$ & + & & \\
\hline L-Threonine & - & 0 & - & & & 7 & - & + & $214,318-323$ \\
\hline L-Leucine & - & 15 & - & - & $\begin{array}{l}101-104,107^{\mathrm{T}} \\
108\end{array}$ & 12 & + & - & $119-128,132$ \\
\hline L-Isoleucine & - & 13 & - & - & $99,102-108$ & 2 & - & + & 319,321 \\
\hline L-Valine & - & 11 & - & - & $\begin{array}{c}98,99,102- \\
106,107^{\mathrm{T}} \\
108,117\end{array}$ & 4 & - & + & $\begin{array}{l}130,319,321 \\
\quad 322\end{array}$ \\
\hline L-Aspartate & - & 9 & - & + & $109,111-118$ & 11 & - & + & $\begin{array}{c}119,121-124, \\
127-132\end{array}$ \\
\hline L-Gutamate & + & 15 & - & - & $\begin{array}{c}102-104,106 \\
107^{\mathrm{T}}, 108\end{array}$ & 14 & + & - & $\begin{array}{c}120,122,216 \\
318-323\end{array}$ \\
\hline L-Lysine & - & 1 & - & + & 99 & 0 & - & & \\
\hline L-Arginine & - & 8 & - & + & $109-116$ & 13 & - & - & $\begin{array}{r}119-124,215^{\mathrm{T}}, \\
216,318,319\end{array}$ \\
\hline L-Ornithine & - & 0 & - & & & 1 & - & + & 214 \\
\hline L-Citrulline & - & 0 & - & & & 2 & + & + & $214,215^{\mathrm{T}}$ \\
\hline L-Histidine & - & 0 & - & & & 11 & - & + & $\begin{array}{l}119,126-132, \\
318,319,323\end{array}$ \\
\hline L-Proline & - & 1 & - & + & 101 & 12 & + & - & $\begin{array}{l}119-125,216, \\
318,319,321\end{array}$ \\
\hline L-Tyrosine & + & $18^{b}$ & - & - & $102,107,117$ & $21^{b}$ & + & - & 122,216 \\
\hline L-Phenylalanine & - & 0 & - & & & 1 & - & + & 214 \\
\hline Hippurate & - & 0 & - & & & 1 & - & + & 214 \\
\hline
\end{tabular}

${ }^{a}$ Strain $217^{\mathrm{T}}$ was the only $A$. nigrifaciens strain tested. The 21 strains of $A$. macleodii tested were strains $98,99,100,101,102,103,104,105$, $106,107^{\mathrm{T}}, 108,109,110,111,112,113,114,115,116,117$, and 118 . The 23 A. haloplanktis strains tested were strains $119,120,121,122,123$, $124,125,126,127,128,129,130,131,132,214,215^{\mathrm{T}}, 216,318,319,320,321,322$, and 323.

${ }^{b}$ More than $80 \%$ of the strains positive.

acid cycle intermediates, and amino acids. The $\mathrm{G}+\mathrm{C}$ content of the DNA is 41.8 to $44.4 \mathrm{~mol} \%$.

The type strain is strain 215 (= ATCC 14393).

The properties which distinguish $A$. macleodii and $A$. haloplanktis from the remaining species of Alteromonas are given in Bergey's Manual of Systematic Bacteriology, 9th ed. (Baumann, Gauthier, and Baumann, in press). Isolates of the marine species Vibrio nigripulchritudo (P. Baumann, A. L. Furniss, and J. V. Lee, in Bergey's Manual of Systematic Bacteriology, 9th ed., in press) and group I-2 (1) produce a blueish black pigment or a brownish black pigment. $V$. nigripulchritudo is differentiated from $A$. nigrifaciens by its ability to ferment carbohydrates. Members of group I-2 are obligately respiratory organisms which are differentiated from $A$. nigrifaciens by their inability to utilize carbohydrates and their $\mathrm{G}+\mathrm{C}$ contents $(61$ to $64 \mathrm{~mol} \%)$. A requirement for approximately $250 \mathrm{mM} \mathrm{NaCl}$ for optimal growth distinguishes $A$. nigrifaciens from most common gram-negative terrestrial microorganisms.

\section{ACKNOWLEDGMENTS}

This study was supported by grant DEB-8205938 from the National Science Foundation, as well as by the Aquaculture Program, University of California Agricultural Experiment Station.

\section{LITERATURE CITED}

1. Baumann, L., P. Baumann, M. Mandel, and R. D. Allen. 1972. Taxonomy of aerobic marine eubacteria. J. Bacteriol. 110:402429.

2. Baumann, P., and L. Baumann. 1981. The marine gram-negative eubacteria: genera Photobacterium, Beneckea, Alteromonas, Pseudomonas, and Alcaligenes, p. 1302-1331. In M. P. Starr, H. Stolp, H. G. Trüper, A. Balows, and H. G. Schlegel (ed.), The prokaryotes. Springer-Verlag, Berlin.

3. Chan, K. Y., L. Baumann, M. M. Garza, and R. D. Allen. 1978. 
Two new species of Alteromonas: Alteromonas espejiana and Alteromonas undina. Int. J. Syst. Bacteriol. 28:217-222.

4. Norton, C. F., and G. E. Jones. 1968. A marine isolate of Pseudomonas nigrifaciens. I. Classification and nutrition. Can. J. Microbiol. 14:1333-1340.

5. Reichelt, J. L., and P. Baumann. 1973. Change of the name Alteromonas marinopraesens (Zobell and Upham) Baumann et al. to Alteromonas haloplanktis (Zobell and Upham) comb. nov. and assignment of strain ATCC 23821 (Pseudomonas enalia) and strain c-A1 of DeVoe and Oginsky to this species. Int. J. Syst. Bacteriol. 23:438-441.

6. Skerman, V. B. D., V. McGowan, and P. H. A. Sneath (ed.) 1980. Approved lists of bacterial names. Int. J. Syst. Bacteriol. 30:225-420.

7. White, A. H. 1940. A bacterial discoloration of print butter. Sci. Agric. 20:638-645. 